\title{
A hinge position distal to the adductor tubercle minimizes the risk of hinge fractures in lateral open wedge distal femoral osteotomy
}

\author{
Philipp W. Winkler ${ }^{1}$. Marco C. Rupp ${ }^{1}$ Patricia M. Lutz ${ }^{1}$ Stephanie Geyer ${ }^{1}$. Philipp Forkel ${ }^{1}$. Andreas B. Imhoff ${ }^{1}$. \\ Matthias J. Feucht ${ }^{1,2}$ (1)
}

Received: 29 June 2020 / Accepted: 14 August 2020 / Published online: 24 August 2020

(c) The Author(s) 2020

\begin{abstract}
Purpose To evaluate the incidence and morphology of medial cortical hinge fractures in lateral open wedge distal femoral osteotomy (LOW-DFO) and to determine a safe zone for the position of the osteotomy hinge to minimize the risk of hinge fractures.

Methods Consecutive patients who underwent LOW-DFO for symptomatic valgus malalignment were screened for eligibility for this retrospective observational cohort study. Demographical and surgical data were collected. The incidence and morphology of medial cortical hinge fractures were evaluated on standard postoperative anterior-posterior knee radiographs. Comprehensive measurements evaluating the osteotomy gap and the position of the osteotomy hinge were taken. Additionally, each osteotomy hinge was assigned to a corresponding sector of a proposed five-sector grid of the distal medial femur. Results A total of 100 patients ( $60 \%$ female) with a mean age of $31 \pm 13$ years were included. The overall incidence of medial cortical hinge fractures was $46 \%$ and three distinct fracture types were identified. The most frequently observed fracture type was extension of the osteotomy gap (76\%), followed by a proximal (20\%) and distal (4\%) course of the fracture line in relation to the hinge. Group comparison (hinge fracture vs. no hinge fracture) showed statistically significant higher values for the height of the osteotomy gap $(p=0.001)$, the wedge angle $(p=0.036)$, and the vertical distance between the hinge and the proximal margin of the adductor tubercle (AT; $p=0.002$ ) in the hinge fracture group. Furthermore, a significantly lower horizontal distance between the hinge and the medial cortical bone $(p=0.036)$ was observed in the hinge fracture group. A statistically significant higher incidence of medial cortical hinge fractures was observed when the position of the osteotomy hinge was proximal compared to distal to the proximal margin of the AT (53\% vs. $27 \% ; p=0.023$ ).

Conclusion Medial cortical hinge fractures in LOW-DFO are a common finding with three distinct fracture types. To minimize the risk of medial cortical hinge fractures, it is recommended to aim for a position of the osteotomy hinge at the level of or distal to the proximal margin of the adductor tubercle.
\end{abstract}

Level of evidence Prognostic study; Level III

Keywords Open wedge $\cdot$ Distal femoral osteotomy $\cdot$ Hinge fracture $\cdot$ Safe zone $\cdot$ Target point $\cdot$ Valgus deformity

\section{Introduction}

The research was performed at the Department for Orthopedic Sports Medicine, Technical University Munich, Germany.

Matthias J. Feucht matthias.feucht@gmx.net

1 Department for Orthopedic Sports Medicine, Klinikum Rechts Der Isar, Technical University of Munich, Ismaninger Str. 22, 81675 Munich, Germany

2 Department of Orthopedics and Trauma Surgery, Medical Center, Faculty of Medicine, Albert-Ludwigs-University of Freiburg, Freiburg, Germany
The correction of valgus malalignment is indicated for the treatment of lateral compartment osteoarthritis (OA) $[2,3$, $6,30,34]$, patellofemoral disorders [5, 11, 27, 32], and in combination with cartilage regenerative or meniscus replacing procedures of the lateral compartment [2]. Varus producing osteotomies are usually performed at the distal femur in a medial closed wedge (MCW) or the lateral open wedge (LOW) technique $[9,36]$.

Good functional, clinical, and radiographic outcomes as well as a reported survivorship of almost $90 \%$ at 5 years and 
more than $70 \%$ at 10 years have made the LOW distal femoral osteotomy (DFO) an attractive technique for the treatment of symptomatic valgus deformity [2, 3, 6, 7, 17, 34]. Encouraging clinical outcomes are supported by a recently published biomechanical study, which has demonstrated the unloading effect of the lateral compartment after LOW-DFO [37]. However, some disadvantages, which are inherently related to the LOW technique, are reported and discredit this technique. The most commonly reported drawbacks include a high rate of reoperations and a considerable number of delayed and non-unions of the osteotomy gap [2, 3, 6, 14, $20,30]$. One reason for non-unions could be a fracture of the medial cortical hinge and the associated reduced axial and torsional stiffness as well as the increased rotational movement across the osteotomy gap for the bone-implant construct $[1,29]$. Therefore, fractures of the medial cortical hinge should be avoided. Safe zones for the position of the osteotomy hinge have been described for medial open wedge high tibial osteotomies (HTO) $[13,24,28]$ and MCW-DFOs $[15,26]$ to minimize the risk of cortical hinge fractures. However, analyses of medial cortical hinge fractures in LOW-DFO and the definition of a safe zone for the osteotomy hinge are missing in the current literature.

Therefore, the primary objective of this retrospective observational cohort study was to evaluate the incidence and morphology of medial cortical hinge fractures in LOWDFO. A further objective was to determine a safe zone for the position of the osteotomy hinge to minimize the risk of medial cortical hinge fractures. It was hypothesized that medial cortical hinge fractures in LOW-DFO are a common finding and that the risk of medial cortical hinge fractures increases with a more proximal position of the osteotomy hinge.

\section{Methods}

Radiographs of 127 consecutive patients who underwent LOW-DFO for symptomatic valgus malalignment between 2015 and 2019 were screened for eligibility for this retrospective observational cohort study. Closed physes, medical records, and postoperative standard anterior to posterior (AP) and lateral knee radiographs were required for inclusion. Patients who had a history of previous osteotomies, fractures, or posttraumatic deformities of the distal femur were excluded from this study. A concomitant correction of a torsional deformity of the distal femur, which is associated with an iatrogenic disruption of the medial cortical bone, led to exclusion of the study. Additionally, patients with a severely malrotated postoperative AP knee radiograph, which was accompanied by a misprojection of the bony landmarks, especially the adductor tubercle (AT) [21], were excluded. Twenty-seven patients did not meet the inclusion criteria (1 posttraumatic deformity, 6 concomitant torsional osteotomies, 20 malrotated radiographs without visualization of the AT). Thus, a total of 100 patients could be included in the present study. A LOW-DFO was performed due to lateral compartment OA or cartilage defects in 50 $(50 \%)$ patients, patellofemoral maltracking associated with patellofemoral instability or OA in $37(37 \%)$ patients, and chronic ligamentous insufficiency in $13(13 \%)$ patients. A review of the medical records was conducted to collect demographical and surgical data. A comprehensive summary of the descriptive statistics of the demographical and surgical data is presented in Table 1 .

Table 1 Descriptive statistics of the demographical and surgical data of the total study group

\begin{tabular}{|c|c|}
\hline Variable & Total study group \\
\hline Number of included patients, $n$ & 100 \\
\hline Age $^{\mathrm{a}}$ (years) & $31.3 \pm 12.7(15-64)$ \\
\hline BMI $\left(\mathrm{kg} / \mathrm{m}^{2}\right)$ & $26.4 \pm 4.9(16.1-35.9)$ \\
\hline \multicolumn{2}{|l|}{ Sex } \\
\hline Male, $n(\%)$ & $40(40 \%)$ \\
\hline Female, $n(\%)$ & $60(60 \%)$ \\
\hline \multicolumn{2}{|l|}{ Laterality } \\
\hline Right, $n(\%)$ & $57(57 \%)$ \\
\hline Left, $n(\%)$ & $43(43 \%)$ \\
\hline \multicolumn{2}{|l|}{ Hinge fracture } \\
\hline Yes, $n(\%)$ & $46(46 \%)$ \\
\hline No, $n(\%)$ & $54(54 \%)$ \\
\hline \multicolumn{2}{|l|}{ Fracture morphology ${ }^{\mathrm{b}}$} \\
\hline Type 1 (extension), $n(\%)$ & $35(76.1 \%)$ \\
\hline Type 2 (distal), $n(\%)$ & $2(4.3 \%)$ \\
\hline Type 3 (proximal), $n(\%)$ & $9(19.6 \%)$ \\
\hline \multicolumn{2}{|l|}{ Concomitant procedures $^{\mathrm{c}}$} \\
\hline None, $n(\%)$ & $48(48 \%)$ \\
\hline HTO-MCW, $n(\%)$ & $4(4 \%)$ \\
\hline Ligament surgery, $n(\%)$ & $36(36 \%)$ \\
\hline PF prosthesis, $n(\%)$ & $7(7 \%)$ \\
\hline Cartilage surgery, $n(\%)$ & $10(10 \%)$ \\
\hline Trochleaplasty, $n(\%)$ & $2(2 \%)$ \\
\hline \multicolumn{2}{|l|}{ Complications } \\
\hline None, $n(\%)$ & $95(95 \%)$ \\
\hline Infection, $n(\%)$ & $2(2 \%)$ \\
\hline Non-union, $n(\%)$ & $2(2 \%)$ \\
\hline Traumatic femur fracture, $n(\%)$ & $1(1 \%)$ \\
\hline
\end{tabular}

Categorical variables are presented as count and percentage; Continuous variables are presented as mean \pm standard deviation (range)

$B M I$, body-mass-index; $H T O-M C W$, high tibial osteotomy medial closed wedge; $P F$, patellofemoral

${ }^{\text {a } A g e ~ a t ~ s u r g e r y ~}$

${ }^{\mathrm{b}}$ Hinge fracture group $(n=46)$

${ }^{\mathrm{c}}$ Total number of patients exceeds 100 (total study group), 7 patients had two concomitant procedures 
Data collection and analysis was performed between December 2019 and April 2020. The approval to conduct this study was granted by the ethical review committee of the Technical University of Munich (No. 6/20S).

\section{Indications and surgical technique}

A comprehensive preoperative analysis of the valgus malalignment based on AP hip-knee-ankle radiographs was followed by a detailed planning of the osteotomy using the medical software mediCAD $\AA$ (mediCAD Hectec GmbH, Altdorf, Deutschland). A postoperative mechanical leg axis crossing the center of the tibial plateau (50\% from medial to lateral) was the desired amount of correction. A step-by-step manual of the performed surgical procedure was previously described in detail [9]. A locking compression plate, PEEKPower ${ }^{\mathrm{TM}}$ Plate (Arthrex Inc., Naples, FL, USA) or TomoFix $^{\text {TM }}$ Plate (DePuy Synthes, Raynham, MA, USA), was used to secure the osteotomy gap. No bone grafting of the osteotomy gap was performed among the included patients. The rehabilitation program started at the first postoperative day and was dependent on the primary diagnosis and the concomitant procedures.

\section{Medial cortical hinge fracture}

A hinge fracture was defined as a disruption of the medial cortical bone. Two observers (P.W.W., M.J.F.) independently assessed each postoperative AP knee radiograph for the presence of a medial cortical hinge fracture. In cases of disagreement, a third observer (M.C.R.) was consulted to achieve consensus. Additionally, the fracture morphology of all medial cortical hinge fractures was evaluated and a classification was established. Each hinge fracture was assigned to the respective fracture type in order to determine the incidence of each type.

\section{Postoperative measurements and hinge position}

For postoperative measurements, standard AP knee radiographs were used, which were acquired on the first or second postoperative day. All measurements were obtained by the main observer (P.W.W.) using the picture archiving and communication system (PACS). For twenty randomly selected patients, measurements were taken twice at an interval of one month by the main observer (P.W.W.) and additionally once by a second observer (M.C.R.) to determine the interand intrarater reliability of measurements.

Measurements were performed as follows: First, the osteotomy hinge was identified and the anatomical axis of the femoral diaphysis was determined. In a detailed analysis, the length (distance "a") and height (distance "b") of the osteotomy gap as well as the wedge angle (angle alpha) and the slope of the osteotomy (angle beta) were measured. Additionally, the position of the osteotomy hinge was quantified by measuring the horizontal distance between the hinge and the medial cortical bone (distance "c") as well as the vertical (distance "d") and horizontal (distance "e") distances between the hinge and the proximal margin of the AT. A detailed description of the performed measurements is shown in Fig. 1.

Furthermore, the position of each osteotomy hinge was assigned to a corresponding sector of an established five-sector grid. Distinct bony landmarks (medial femoral cortical bone, femoral condyles, AT) were used to define three rows (I, II, III) and two columns (M, L) of the grid. According to the respective row and column, the sectors were termed IL, IIL, IIIL, IIM, IIIM. Given the definition of the grid, there is no sector IM. A detailed description of the five-sector grid is presented in Fig. 2. The allocation of the hinge position to the corresponding sector was performed by two observers (P.W.W., M.J.F) in agreement with each other.

ICC values of measurements indicated good to excellent intrarater reliability (distance ,a “, 0.997; distance "b", 0.883; distance "c", 0.966; distance "d", 0.982; distance "e", 0.983; angle alpha, 0.786; angle beta, 0.945) and moderate to excellent interrater reliability (distance " $a$ ", 0.788; distance "b", 0.875; distance "c", 0.681; distance "d", 0.952; distance "e", 0.819; angle alpha, 0.637; angle beta, 0.959).

\section{Statistical analysis}

An a priori power analysis, performed with $\mathrm{G}^{*}$ Power (Erdfelder, Faul, Buchner, Lang, HHU Düsseldorf, Düsseldorf, Germany) [8], revealed a total sample size of 90 subjects to detect a difference of $1 \mathrm{~mm}$ of the hinge position at an assumed effect size of 0.6 in order to achieve a statistical power of 0.8 .

SPSS software version 26.0 (IBM-SPSS, New York, USA) was used for statistical analysis. The level of significance was defined as $p<0.05$. Categorical variables were reported as count and percentages. Continuous variables were calculated as mean \pm standard deviation. Normal distribution of continuous variables was assessed by the Shapiro-Wilk-Test. Group comparison of categorical variables was performed with the Chi-square test or the Fisher's exact test, as appropriate. Group comparison of continuous variables was performed with the Mann-Whitney $U$ test or an unpaired $t$ test, as appropriate.

A binary logistic regression was performed to determine the odds of sustaining a medial cortical hinge fracture. The event "medial cortical hinge fracture" (no vs. yes) was defined as the dependent variable. Independent variables which describe the hinge position and demonstrated a significant difference $(p<0.05)$ between the two groups (hinge 


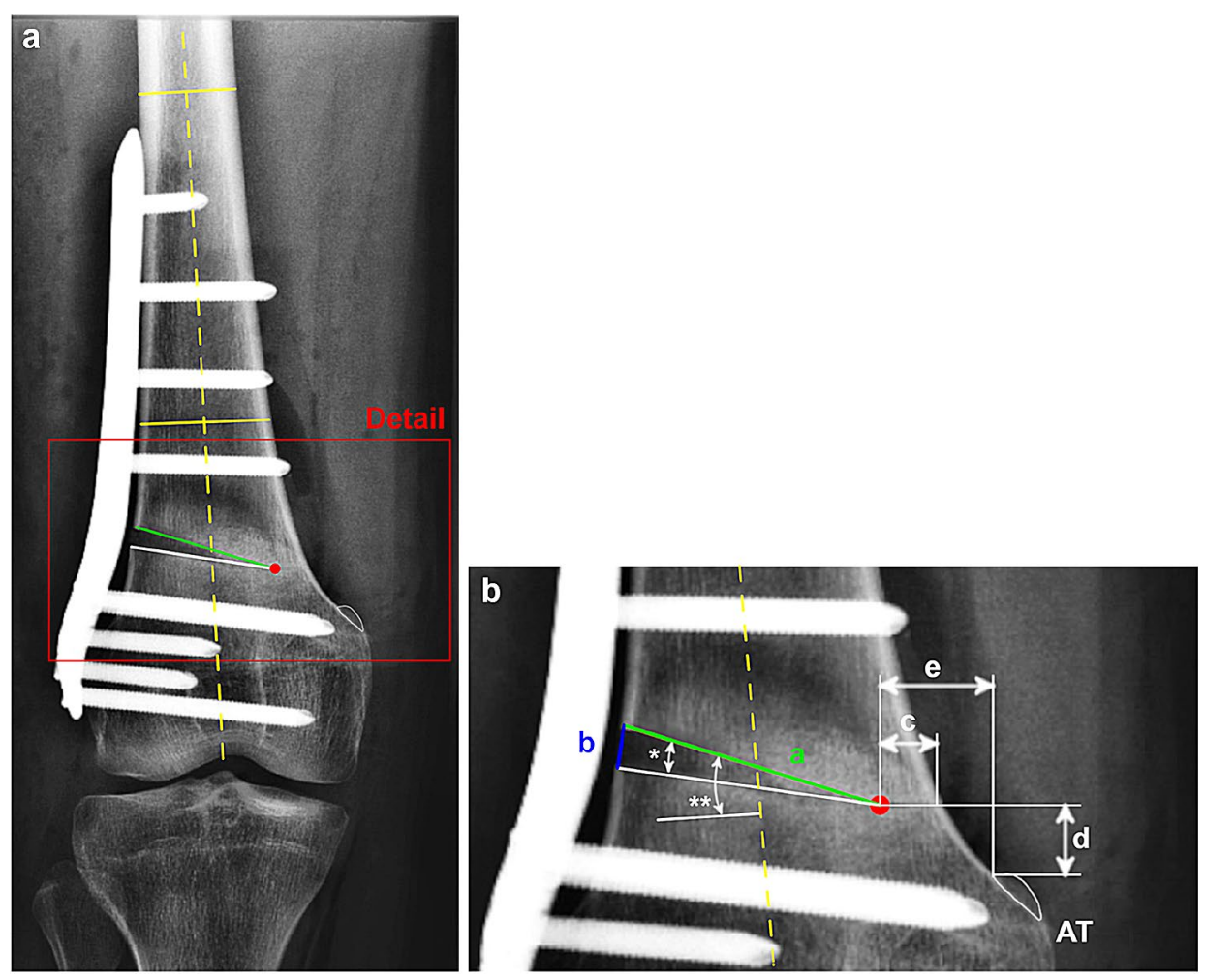

Fig. 1 Postoperative radiographic measurements. a Standard anterior-posterior radiograph of a right knee after lateral open wedge distal femoral osteotomy using a TomoFix ${ }^{\mathrm{TM}}$ (DePuy Synthes, Raynham, MA, USA) locking compression plate. b Detailed view of the osteotomy gap with the corresponding measurements. Red dot, osteotomy hinge; $A T$ adductor tubercle (encircled in white); Yellow solid lines, medial-lateral connection of the femoral cortical bone; Yellow dashed line, anatomical axis of the femoral diaphysis, defined as the line connecting the midpoints of the two yellow solid lines; Green line (distance "a"), length of the osteotomy gap; Blue line (distance

fracture vs. no hinge fracture) in univariate analysis, were used as the covariates.

\section{Results}

\section{Incidence and fracture morphology}

The overall incidence of medial cortical hinge fractures in LOW-DFO was $46 \%$. Three different fracture types could be observed (Fig. 3). The most frequently observed fracture morphology was type 1 (extension, 76\%), followed by type 3 (proximal, 20\%) and type 2 (distal, $4 \%$ ).

\section{Hinge fracture versus no hinge fracture}

A detailed summary of the group comparison is shown in Table 2. For postoperative measurements, a statistically "b"), height of the osteotomy gap; Distance "c", horizontal distance between the medial cortical bone and the osteotomy hinge; Distance "d", vertical distance between the proximal margin of the AT and the osteotomy hinge; Distance "e", horizontal distance between the proximal lateral margin of the AT and the osteotomy hinge; *(angle alpha), wedge angle between the proximal and distal osteotomy plane; **(angle beta), slope of the osteotomy defined as the angle between a perpendicular line to the anatomical axis of the femur and the proximal osteotomy plane

significant difference between the two groups could be observed for the height of the osteotomy gap (distance " $\mathrm{b}$ ", $p=0.001$ ) and the wedge angle (alpha, $p=0.036$ ). Considering the position of the osteotomy hinge, there was a statistically significant difference between the groups for the horizontal distance to the medial cortical bone (distance "c", $p=0.036$ ) and the vertical distance to the proximal margin of the AT (distance "d", $p=0.002$ ). Additionally, a statistically significant difference for the sector-based hinge position ( $p=0.006$; Table 3$)$ and for the hinge position in relation to the proximal margin of the AT (proximal vs. distal; $p=0.023$ ) between the hinge fracture group and the no hinge fracture group could be observed (Fig. 4). The incidence of medial cortical hinge fractures was 53\% when the osteotomy hinge was located proximal to the AT and $27 \%$ when its location was at the level of or distal to the proximal margin of the AT. 


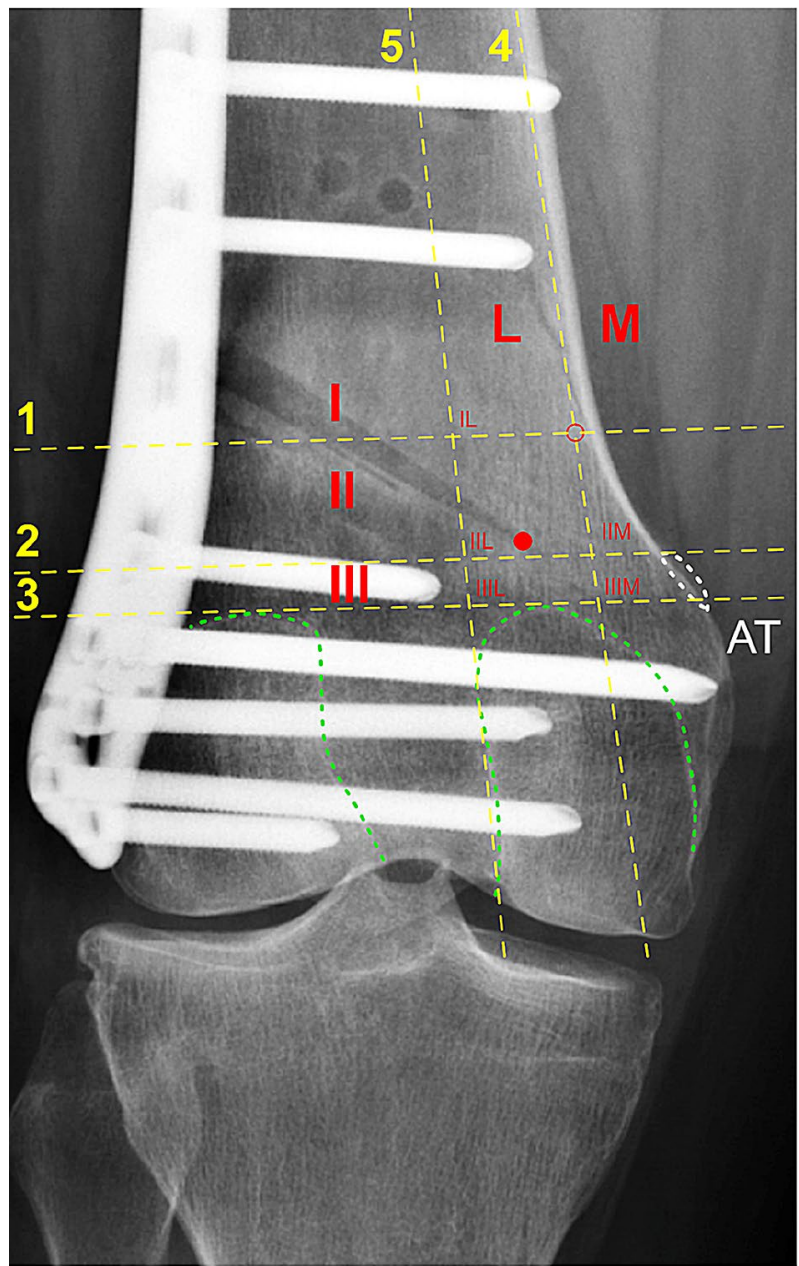

Fig. 2 Five-sector grid. $A T$, adductor tubercle (encircled in white); $L$, lateral; $M$, medial; $I$, row 1; II, row 2; III, row 3; Green dashed lines, posterior part of the medial and lateral femoral condyle; Red dot, osteotomy hinge; Red circle, inflection point, defined as the point at which the distance between the medial cortical bone and Line 4 reaches $2 \mathrm{~mm}$; Line 5 , tangential to the lateral facet of the medial femoral condyle; Line 4, tangential to the medial femoral cortical bone; Line 3, tangential to the apices of the posterior part of the medial and lateral femoral condyle; Line 2, parallel to Line 3 and crossing the proximal part of the AT; Line 1, parallel to Line 3 and crossing the inflection point

A binary logistic regression model for the occurrence of the event "medial cortical hinge fracture" showed statistical significance for the model itself $(p=0.001)$ as well as for the height of the osteotomy gap (distance "b"; $p=0.036$ ), and for the vertical distance to the proximal margin of the AT (distance "d"; $p=0.032$ ). An increase of the osteotomy height (distance "b") and the vertical distance to the AT (distance "d") by $1 \mathrm{~mm}$, increases the odds of sustaining a medial cortical hinge fracture by $41 \%$ and $11 \%$, respectively (Table 4).

\section{Discussion}

The present study provides evidence for two major findings; first, with an incidence of $46 \%$, a medial cortical hinge fracture in LOW-DFO is a common finding, which presents itself in three distinct types. In spite of the high incidence, there is no difference in the complication rate for patients with and without a medial cortical hinge fracture. Second, a more proximal position of the osteotomy hinge leads to a higher incidence and increased odds of medial cortical hinge fractures. It is therefore recommended to aim for a position of the osteotomy hinge in sector IIIL or IIIM (at the level of or distal to the proximal margin of the AT) according to the presented five-sector grid when performing a LOW-DFO.

Fractures of the lateral cortical hinge after medial open wedge HTO have been extensively investigated. With an incidence of $18-50 \%$ [4, 12, 16, 18, 19, 22-25, 28, 33], lateral cortical hinge fractures in HTO are a common finding and have been associated with the occurrence of nonunions of the osteotomy gap [4, 12, 23]. Similar results have been shown for DFO. The incidence of lateral/medial cortical hinge fractures is reported to be almost $48 \%$ and $39 \%$ for the MCW [10] and the LOW [20] technique, respectively. This is in accordance with an overall incidence of $46 \%$ of medial cortical hinge fractures in LOWDFOs in the present study. Non-unions of the osteotomy gap are dreaded complications of LOW-DFO [2, 3, 6, 14, $20,30]$ and may be attributed to medial cortical hinge fractures and the associated reduced axial and torsional stiffness as well as the increased rotational movement across the osteotomy gap for the bone-implant construct $[1,29]$. However, despite the high incidence of hinge fractures in the present study, the complication rate was only $5 \%$ and showed no difference between patients with and without a medial cortical hinge fracture.

In 2012, Takeuchi et al. [33] proposed a classification for lateral cortical hinge fractures in medial open wedge HTO. In a retrospective analysis of 104 medial open wedge HTOs, 26 (25\%) fractures of the lateral cortical hinge with three different fracture types were observed [33]. Similarly, three distinct types of medial cortical hinge fractures have been observed in LOW-DFO in the present study (Fig. 3). With an incidence of $76 \%$ among the hinge fractures, fractures in extension of the osteotomy gap (type 1) were most common. According to the distribution of fracture types for the present study, fractures in extension of the osteotomy gap are also most frequently observed in medial open wedge HTOs [12, 18, 24, 25, 28, 33].

Since distinct fracture types (Takeuchi type 2 and type 3) after medial open wedge HTOs are associated with an 

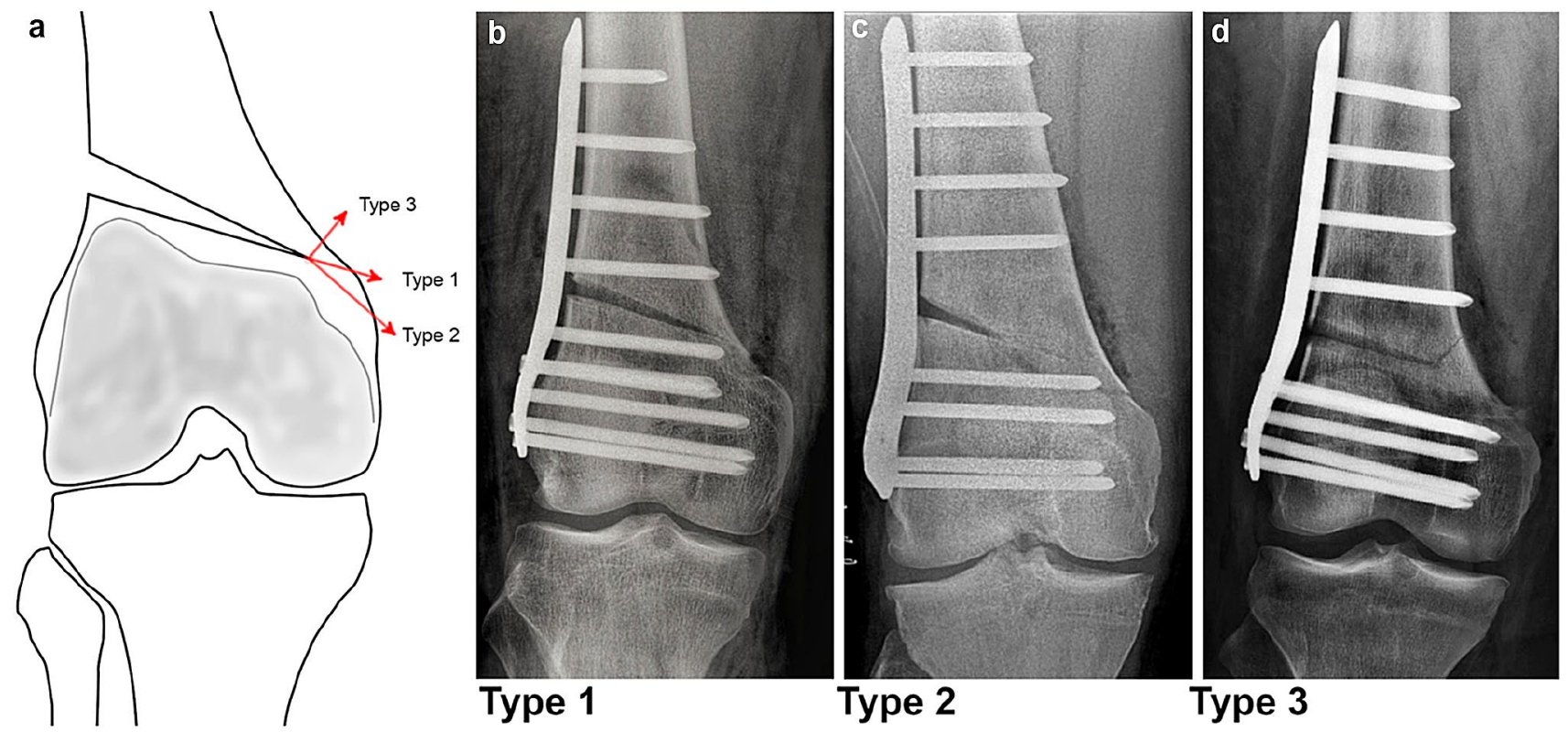

Type 1

Type 2

Type 3

Fig. 3 Morphology and classification of medial cortical hinge fractures. a Schematic illustration of the three different fracture types. b Type 1 fracture, extension of the osteotomy plane. $\mathbf{c}$ Type 2 fracture, distal to the osteotomy hinge. $\mathbf{d}$ Type 3 fracture, proximal to the osteotomy hinge

increased complication rate [12, 25, 33], many efforts have been made to establish a safe zone for the position of the osteotomy hinge in order to reduce the risk of hinge fractures [13, 24, 28]. A recently published cadaveric analysis of lateral cortical hinge fractures in MCW-DFO concluded that the ideal position of the osteotomy hinge is at the upper border of the lateral femoral condyle to minimize the risk of unstable lateral cortical hinge fractures [15]. This conclusion was supported by two main findings. First, the upper border of the lateral femoral condyle is located within the femoral attachment site of the lateral gastrocnemius head. As a result, the femoral attachment of lateral gastrocnemius head acts as a soft tissue stabilizer of the osteotomy hinge [15]. Second, an area of low bone density was observed in the region of the upper border of the lateral femoral condyle. The authors assumed that this area tolerates increased plastic deformation and thus reduces the risk of lateral cortical hinge fractures [15]. A second biomechanical study showed similar results with a higher incidence and increased propensity for instability of lateral cortical hinge fractures in MCW-DFO with a supracondylar hinge position compared to a condylar hinge position [26]. Consistent to the proposed safe zones for the MCW technique, a condylar hinge position at the level of or distal to the proximal margin of the AT has been shown to reduce the odds of hinge fractures for the LOW technique, as demonstrated in the present study. Whether or not additional soft tissue stabilizers and certain bone density patterns at the distal medial femur contribute to the formation of medial cortical hinge fractures has not been investigated yet and therefore remains a subject of future research.

A biplanar technique for LOW-DFO is recommended to achieve a position of the osteotomy hinge at the level of or distal to the proximal margin of the AT without harming the trochlea groove [9]. Besides the ability of a substantially more distal level of the axial osteotomy, the biplanar technique has several more advantages. One study showed an increased torsional stiffness and reduced rotational movement across the osteotomy gap for the biplanar technique compared to the uniplanar technique, especially when a disruption of the medial cortical bone was simulated [29]. Another important benefit of the biplanar technique is the increased bone-surface area, which is believed to improve bone healing [35]. Osseous consolidation is further supported by the improved healing potential of the metaphyseal compared to the diaphyseal bone [31].

There are some limitations of the present study. Given the absence of clinical outcomes, it is not possible to make a conclusion on whether a fracture of the medial cortical hinge affects the functional and clinical outcomes. Since this study was conducted to analyze the incidence and morphology of medial cortical hinge fractures as well as to describe a safe zone for the osteotomy hinge, collecting and reporting clinical data would have been beyond the scope of this study. Furthermore, the incidence of medial cortical hinge fractures in LOW-DFO was assessed by simple postoperative AP radiographs. However, previous studies investigating 
Table 2 Group comparison (hinge fracture vs. no hinge fracture)

\begin{tabular}{|c|c|c|c|}
\hline \multirow[t]{2}{*}{ Variable } & \multicolumn{2}{|c|}{ Hinge fracture } & \multirow[t]{2}{*}{$p$ value } \\
\hline & No & Yes & \\
\hline Number of patients, $n$ & 54 & 46 & - \\
\hline $\operatorname{Age}^{\mathrm{a}}$ (years) & $28.7 \pm 10.8$ & $34.3 \pm 14.2$ & n.s \\
\hline BMI $\left(\mathrm{kg} / \mathrm{m}^{2}\right)$ & $26.7 \pm 4.9$ & $25.9 \pm 5.0$ & n.s \\
\hline \multicolumn{4}{|l|}{ Sex } \\
\hline Male, $n(\%)$ & $19(35.2 \%)$ & $21(45.7 \%)$ & \multirow[t]{2}{*}{ n.s } \\
\hline Female, $n(\%)$ & $35(64.8 \%)$ & $25(54.3 \%)$ & \\
\hline \multicolumn{3}{|l|}{ Laterality } & \multirow[t]{3}{*}{ n.s } \\
\hline Right, $n(\%)$ & $30(55.6 \%)$ & $27(58.7 \%)$ & \\
\hline Left, $n(\%)$ & $24(44.4 \%)$ & $19(41.3 \%)$ & \\
\hline Distance a $[\mathrm{mm}]$ & $55.8 \pm 12.9$ & $53.1 \pm 12.8$ & n.s \\
\hline Distance b [mm] & $5.6 \pm 2.2$ & $6.9 \pm 1.9$ & $0.001 *$ \\
\hline Distance c [mm] & $8.8 \pm 4.2$ & $7.2 \pm 5.1$ & $0.036^{*}$ \\
\hline Distance d [mm] & $4.9 \pm 8.5$ & $10.6 \pm 9.4$ & $0.002 *$ \\
\hline Distance e [mm] & $14.5 \pm 6.4$ & $17.0 \pm 7.7$ & n.s \\
\hline Alpha $\left[{ }^{\circ}\right]$ & $6.1 \pm 2.6$ & $7.1 \pm 2.6$ & $0.036^{*}$ \\
\hline Beta $\left[{ }^{\circ}\right]$ & $25.5 \pm 5.8$ & $23.6 \pm 7.0$ & n.s \\
\hline \multicolumn{3}{|l|}{ Concomitant procedures $^{\mathrm{b}}$} & \multirow[t]{7}{*}{ n.s } \\
\hline None, $n(\%)$ & $30(51.7 \%)$ & $18(36.7 \%)$ & \\
\hline HTO-MCW, $n(\%)$ & $2(3.4 \%)$ & $2(4.1 \%)$ & \\
\hline Ligament surgery, $n(\%)$ & $16(27.6 \%)$ & $20(40.8 \%)$ & \\
\hline PF prosthesis, $n(\%)$ & $2(3.4 \%)$ & $5(10.2 \%)$ & \\
\hline Cartilage surgery, $n(\%)$ & $6(10.3 \%)$ & $4(8.2 \%)$ & \\
\hline Trochleaplasty, $n(\%)$ & $2(3.4 \%)$ & $0(0 \%)$ & \\
\hline \multicolumn{4}{|l|}{ Complications } \\
\hline None, $n(\%)$ & $52(96.3 \%)$ & $43(93.5 \%)$ & \multirow[t]{4}{*}{ n.s } \\
\hline Infection, $n(\%)$ & $0(0 \%)$ & $2(4.3 \%)$ & \\
\hline Non-union, $n(\%)$ & $2(3.7 \%)$ & $0(0 \%)$ & \\
\hline Traumatic femur fracture, $n(\%)$ & $0(0 \%)$ & $1(2.2 \%)$ & \\
\hline
\end{tabular}

Categorical variables are presented as count and percentage; Continuous variables are presented as mean \pm standard deviation; Positive values of distance " $\mathrm{d}$ " indicated a hinge position proximal to the adductor tubercle (AT), while negative values indicated a hinge position distal to the proximal margin of the AT

$B M I$, body-mass-index; HTO-MCW, high tibial osteotomy medial closed wedge; n.s., non-significant; $P F$, patellofemoral

${ }^{\mathrm{a}}$ Age at surgery

${ }^{\mathrm{b}}$ Total number of patients exceeds 100 (total study group), 7 patients had two concomitant procedures

*Statistically significant difference between groups (level of significance, $p<0.05$ ) lateral cortical hinge fractures in medial open wedge HTO showed that the incidence of hinge fractures is even higher when assessed by computed tomography (CT) $[18,19]$. To avoid unnecessary radiation, postoperative CT scans are not routinely acquired, which is why simple radiographs were used for the present study. The proposed five-sector grid may be affected by a malrotation of the knee during image acquisition, which can falsify a reliable assignment of the osteotomy hinge to the corresponding sector. It is important to keep this in mind, especially when the five-sector grid is applied during surgery.

\section{Conclusion}

Medial cortical hinge fractures in LOW-DFO are a common finding with three distinct fracture types. The most frequently observed fracture type was an extension of the osteotomy plane (type 1, 76\%). To minimize the risk of medial cortical hinge fractures, it is recommended to aim for a position of the osteotomy hinge at the level of or distal to the proximal margin of the adductor tubercle. 
Table 3 Group comparison of the sector-based hinge position (IL, IIL, IIIL, IIM, IIIM)
Fig. 4 Group comparison of the hinge position (hinge fracture vs. no hinge fracture). Percentages are given for the respective sector; $A T$, adductor tubercle; *statistically significant more hinge fractures for a position of the osteotomy hinge proximal to the AT compared to a position distal to the proximal margin of the AT (level of significance, $p<0.05)$

\begin{tabular}{llllllll}
\hline Variable & \multicolumn{2}{l}{ Sector-based hinge position } & & \multirow{2}{l}{$p$ value } \\
\cline { 2 - 6 } \cline { 4 - 5 } & IL & IIL & IIIL & IIM & IIIM & \\
\hline Number of patients, $n$ & $6(6.0 \%)$ & $43(43.0 \%)$ & $8(8.0 \%)$ & $25(25.0 \%)$ & $18(18.0 \%)$ & - \\
Hinge fracture & & & & & & \\
Yes, $n(\%)$ & $5(83.3 \%)$ & $17(39.5 \%)$ & $1(12.5 \%)$ & $17(68.0 \%)$ & $6(33.3 \%)$ & $0.006^{*}$ \\
No, $n(\%)$ & $1(16.7 \%)$ & $26(60.5 \%)$ & $7(87.5 \%)$ & $8(32.0 \%)$ & $12(66.7 \%)$ & \\
Fracture morphology & & & & & & \\
Type 1 (extension), $n(\%)$ & $5(100 \%)$ & $8(47.1 \%)$ & $0(0.0 \%)$ & $16(94.1 \%)$ & $6(100 \%)$ & \multirow{2}{*}{ n.s } \\
Type 2 (distal), $n(\%)$ & $0(0.0 \%)$ & $1(5.9 \%)$ & $0(0.0 \%)$ & $1(5.9 \%)$ & $0(0.0 \%)$ & \\
Type 3 (proximal), $n(\%)$ & $0(0.0 \%)$ & $8(47.1 \%)$ & $1(100 \%)$ & $0(0.0 \%)$ & $0(0.0 \%)$ & \\
\hline
\end{tabular}

Categorical variables are presented as count and percentage

n.s. non-significant

${ }^{\text {a }}$ Hinge fracture group $(n=46)$

*Statistically significant difference between groups (level of significance, $p<0.05$ )

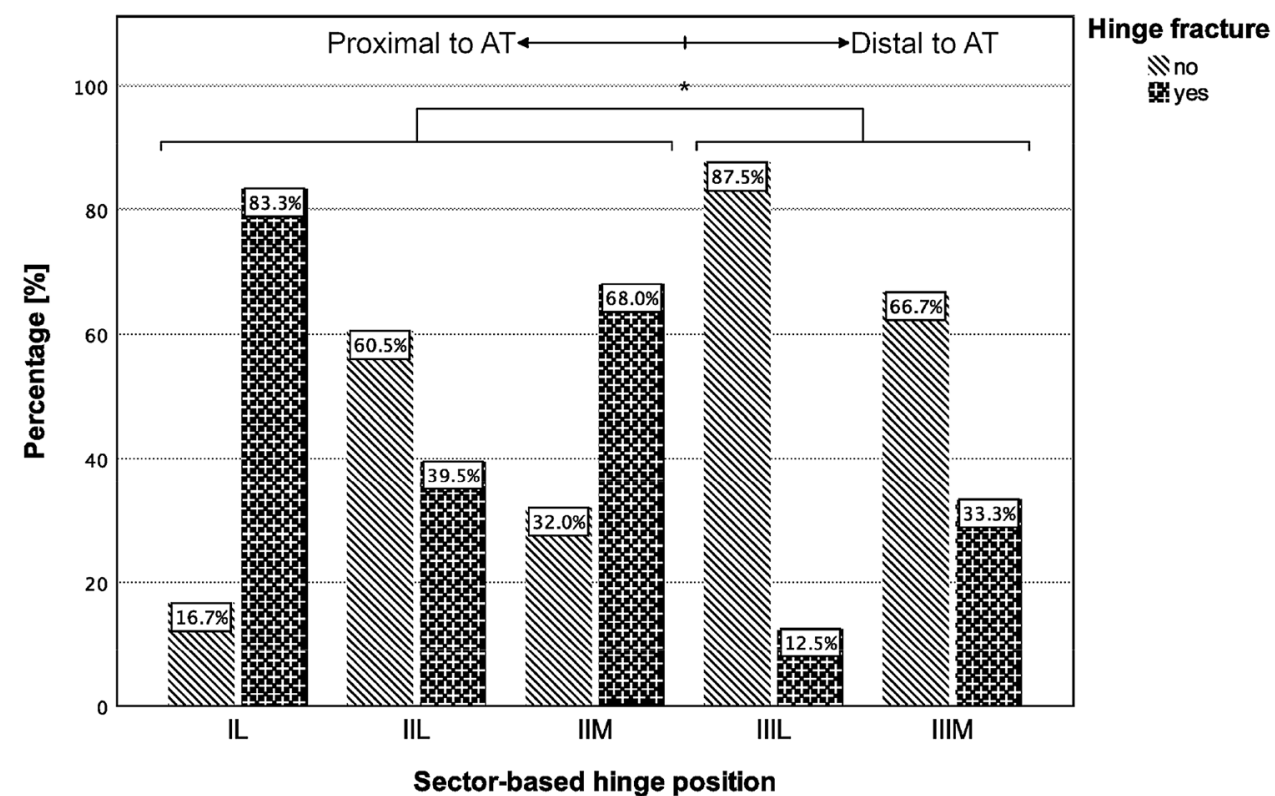

Table 4 Binary logistic regression model for the event "hinge fracture (no vs. yes)"

\begin{tabular}{lllll}
\hline Variable & Reference & $p$ value & OR & $95 \%$ CI \\
\hline $\begin{array}{l}\text { Sector-based hinge posi- } \\
\text { tion }\end{array}$ & & & & \\
IL & IIL vs IL & n.s & 4.28 & $0.36-51.10$ \\
IIL & Ref & Ref & Ref & Ref \\
IIIL & IIL vs IIIL & n.s & 1.27 & $0.08-20.10$ \\
IIM & IIL vs IIM & n.s & 3.10 & $0.94-10.27$ \\
IIIM & IIL vs IIIM & n.s & 2.63 & $0.36-19.18$ \\
Distance b & - & $0.036^{*}$ & 1.41 & $1.02-1.94$ \\
Distance c & - & n.s & 0.97 & $0.86-1.10$ \\
Distance d & - & $0.032^{*}$ & 1.11 & $1.01-1.21$ \\
Angle alpha & - & n.s & 0.94 & $0.73-1.21$ \\
\hline
\end{tabular}

For the categorical variable, sector-based hinge position, sector IIL was considered as the reference sector, since the osteotomy hinge was localized most frequently in sector IIL. The distances and angles were entered in millimeters and degrees, respectively

$C I$, confidence interval; $n . s$., non-significant; $O R$, odds ratio; Ref, reference

* Statistically significant (level of significance, $p<0.05$ ) 
Author contributions All listed authors have contributed substantially to this work: PWW, MJF, PF, ABI for the study conception and design; PWW, PML, MCR and SG for the data collection; PWW and MJF for the data analysis; PWW, MJF, PF and ABI for the data interpretation; PWW, PML, MCR and SG for the drafting of the manuscript, the figures, and the literature research; PWW, MJF, PML, MCR, SG, PF and $\mathrm{ABI}$ for critical revising the manuscript.

Funding No funding was received for conducting this study. Open Access funding provided by Projekt DEAL.

\section{Compliance with ethical standards}

Conflict of interest Andreas B. Imhoff is a consultant for Arthrosurface and Medi Bayreuth and receives royalties from Arthrex and Arthrosurface.

Ethical approval This study was approved by the Ethics Committee of the Technical University of Munich (Nr.: 6/20 S).

Open Access This article is licensed under a Creative Commons Attribution 4.0 International License, which permits use, sharing, adaptation, distribution and reproduction in any medium or format, as long as you give appropriate credit to the original author(s) and the source, provide a link to the Creative Commons licence, and indicate if changes were made. The images or other third party material in this article are included in the article's Creative Commons licence, unless indicated otherwise in a credit line to the material. If material is not included in the article's Creative Commons licence and your intended use is not permitted by statutory regulation or exceeds the permitted use, you will need to obtain permission directly from the copyright holder. To view a copy of this licence, visit http://creativecommons.org/licenses/by/4.0/.

\section{References}

1. Batista BB, Volpon JB, Shimano AC, Kfuri M Jr (2015) Varization open-wedge osteotomy of the distal femur: comparison between locking plate and angle blade plate constructs. Knee Surg Sports Traumatol Arthrosc 23:2202-2207

2. Cameron JI, McCauley JC, Kermanshahi AY, Bugbee WD (2015) Lateral Opening-wedge distal femoral osteotomy: pain relief, functional improvement, and survivorship at 5 years. Clin Orthop Relat Res 473:2009-2015

3. Dewilde TR, Dauw J, Vandenneucker H, Bellemans J (2013) Opening wedge distal femoral varus osteotomy using the Puddu plate and calcium phosphate bone cement. Knee Surg Sports Traumatol Arthrosc 21:249-254

4. Dexel J, Fritzsche H, Beyer F, Harman MK, Lützner J (2017) Open-wedge high tibial osteotomy: incidence of lateral cortex fractures and influence of fixation device on osteotomy healing. Knee Surg Sports Traumatol Arthrosc 25:832-837

5. Dickschas J, Ferner F, Lutter C, Gelse K, Harrer J, Strecker W (2018) Patellofemoral dysbalance and genua valga: outcome after femoral varisation osteotomies. Arch Orthop Trauma Surg 138:19-25

6. Ekeland A, Nerhus TK, Dimmen S, Heir S (2016) Good functional results of distal femoral opening-wedge osteotomy of knees with lateral osteoarthritis. Knee Surg Sports Traumatol Arthrosc 24:1702-1709

7. Elattar O, Swarup I, Lam A, Nguyen J, Fragomen A, Rozbruch SR (2017) Open wedge distal femoral osteotomy: accuracy of correction and patient outcomes. HSS J 13:128-135
8. Faul F, Erdfelder E, Lang AG, Buchner A (2007) G*Power 3: a flexible statistical power analysis program for the social, behavioral, and biomedical sciences. Behav Res Methods 39:175-191

9. Feucht MJ, Mehl J, Forkel P, Imhoff AB, Hinterwimmer S (2017) Distal femoral osteotomy using a lateral opening wedge technique. Oper Orthop Traumatol 29:320-329

10. Forkel P, Achtnich A, Metzlaff S, Zantop T, Petersen W (2015) Midterm results following medial closed wedge distal femoral osteotomy stabilized with a locking internal fixation device. Knee Surg Sports Traumatol Arthrosc 23:2061-2067

11. Frings J, Krause M, Akoto R, Wohlmuth P, Frosch KH (2018) Combined distal femoral osteotomy (DFO) in genu valgum leads to reliable patellar stabilization and an improvement in knee function. Knee Surg Sports Traumatol Arthrosc 26:3572-3581

12. Goshima K, Sawaguchi T, Shigemoto K, Iwai S, Nakanishi A, Inoue D et al (2019) Large opening gaps, unstable hinge fractures, and osteotomy line below the safe zone cause delayed bone healing after open-wedge high tibial osteotomy. Knee Surg Sports Traumatol Arthrosc 27:1291-1298

13. Han SB, Lee DH, Shetty GM, Chae DJ, Song JG, Nha KW (2013) A "safe zone" in medial open-wedge high tibia osteotomy to prevent lateral cortex fracture. Knee Surg Sports Traumatol Arthrosc 21:90-95

14. Jacobi M, Wahl P, Bouaicha S, Jakob RP, Gautier E (2011) Distal femoral varus osteotomy: problems associated with the lateral open-wedge technique. Arch Orthop Trauma Surg 131:725-728

15. Kim TW, Lee MC, Cho JH, Kim JS, Lee YS (2019) The ideal location of the lateral hinge in medial closing wedge osteotomy of the distal femur: analysis of soft tissue coverage and bone density. Am J Sports Med 47:2945-2951

16. Kim TW, Lee SH, Lee JY, Lee YS (2019) Effect of fibular height and lateral tibial condylar geometry on lateral cortical hinge fracture in open wedge high tibial osteotomy. Arthroscopy 35:1713-1720

17. Kolb A, Isak V, Hobusch GM, Chiari C, Windhager R (2019) Distal femoral varus osteotomy: results of the lateral open-wedge technique without bone grafting. Int Orthop 43:2315-2322

18. Lee OS, Lee YS (2018) Diagnostic value of computed tomography and risk factors for lateral hinge fracture in the open wedge high tibial osteotomy. Arthroscopy 34:1032-1043

19. Lee SS, Celik H, Lee DH (2018) Predictive factors for and detection of lateral hinge fractures following open wedge high tibial osteotomy: plain radiography versus computed tomography. Arthroscopy 34:3073-3079

20. Liska F, Voss A, Imhoff FB, Willinger L, Imhoff AB (2018) Nonunion and delayed union in lateral open wedge distal femoral osteotomies-a legitimate concern? Int Orthop 42:9-15

21. Liu KL, Wang CC, Chen IH, Chang CM, Wu WT, Yeh KT (2018) Radiographic morphology and method for localization of the adductor tubercle on anterior-posterior knee radiographs. J Knee Surg 31:747-753

22. Martin R, Birmingham TB, Willits K, Litchfield R, Lebel ME, Giffin JR (2014) Adverse event rates and classifications in medial opening wedge high tibial osteotomy. Am J Sports Med 42:1118-1126

23. Meidinger G, Imhoff AB, Paul J, Kirchhoff C, Sauerschnig M, Hinterwimmer S (2011) May smokers and overweight patients be treated with a medial open-wedge HTO? Risk factors for nonunion. Knee Surg Sports Traumatol Arthrosc 19:333-339

24. Nakamura R, Komatsu N, Fujita K, Kuroda K, Takahashi M, Omi $\mathrm{R}$ et al (2017) Appropriate hinge position for prevention of unstable lateral hinge fracture in open wedge high tibial osteotomy. Bone Joint J 99b:1313-1318

25. Nakamura R, Komatsu N, Murao T, Okamoto Y, Nakamura S, Fujita K et al (2015) The validity of the classification for lateral 
hinge fractures in open wedge high tibial osteotomy. Bone Joint J 97b:1226-1231

26. Nha KW, Chang YS, Shon OJ, Shim BJ, Lee JS, Song JS et al (2019) Where is the target point to prevent cortical hinge fracture in medial closing-wedge distal femoral varus osteotomy? J Knee Surg 32:274-279

27. Nha KW, Ha Y, Oh S, Nikumbha VP, Kwon SK, Shin WJ et al (2018) Surgical treatment with closing-wedge distal femoral osteotomy for recurrent patellar dislocation with genu valgum. Am J Sports Med 46:1632-1640

28. Ogawa H, Matsumoto K, Akiyama H (2017) The prevention of a lateral hinge fracture as a complication of a medial opening wedge high tibial osteotomy: a case control study. Bone Joint $\mathrm{J}$ $99 \mathrm{~b}: 887-893$

29. Pietsch M, Hochegger M, Winkler M, Sandriesser S, Freude T, Augat P (2019) Opening-wedge osteotomies of the distal femur: minor advantages for a biplanar compared to a uniplanar technique. Knee Surg Sports Traumatol Arthrosc 27:2375-2384

30. Saithna A, Kundra R, Getgood A, Spalding T (2014) Opening wedge distal femoral varus osteotomy for lateral compartment osteoarthritis in the valgus knee. Knee 21:172-175

31. Simon MH, Grünwald L, Schenke M, Dickschas J, Strecker W (2020) Corrective osteotomies of femur and tibia: which factors influence bone healing? Arch Orthop Trauma Surg 140:303-311
32. Swarup I, Elattar O, Rozbruch SR (2017) Patellar instability treated with distal femoral osteotomy. Knee 24:608-614

33. Takeuchi R, Ishikawa H, Kumagai K, Yamaguchi Y, Chiba N, Akamatsu Y et al (2012) Fractures around the lateral cortical hinge after a medial opening-wedge high tibial osteotomy: a new classification of lateral hinge fracture. Arthroscopy 28:85-94

34. Thein R, Bronak S, Thein R, Haviv B (2012) Distal femoral osteotomy for valgus arthritic knees. J Orthop Sci 17:745-749

35. van Heerwaarden R, Najfeld M, Brinkman M, Seil R, Madry H, Pape D (2013) Wedge volume and osteotomy surface depend on surgical technique for distal femoral osteotomy. Knee Surg Sports Traumatol Arthrosc 21:206-212

36. Wylie JD, Maak TG (2016) Medial closing-wedge distal femoral osteotomy for genu valgum with lateral compartment disease. Arthrosc Tech 5:e1357-e1366

37. Wylie JD, Scheiderer B, Obopilwe E, Baldino JB, Pavano C, Macken CJ et al (2018) The effect of lateral opening wedge distal femoral varus osteotomy on tibiofemoral contact mechanics through knee flexion. Am J Sports Med 46:3237-3244

Publisher's Note Springer Nature remains neutral with regard to jurisdictional claims in published maps and institutional affiliations. 